\title{
Disons
}

Revista Educación

ISSN: 0379-7082

ISSN: 2215-2644

revedu@gmail.com

Universidad de Costa Rica

Costa Rica

\section{Significados de reflexión sobre la acción docente en el estudiantado y sus formadores en una universidad chilena}

Nocetti de la Barra, Alejandra; Medina-Moya, José Luis

Significados de reflexión sobre la acción docente en el estudiantado y sus formadores en una universidad chilena Revista Educación, vol. 43, núm. 1, 2019

Universidad de Costa Rica, Costa Rica

Disponible en: http://www.redalyc.org/articulo.oa?id=44057415010

DOI: https://doi.org/10.15517/revedu.v43i1.28041

Esta obra está bajo una Licencia Creative Commons Atribución-NoComercial-SinDerivar 3.0 Internacional. 


\title{
Significados de reflexión sobre la acción docente en el estudiantado y sus formadores
} en una universidad chilena

\author{
The significance of education practices and their effect on students at a Chilean University
}

Alejandra Nocetti de la Barra

DOI: https://doi.org/10.15517/revedu.v43i1.28041

Universidad Católica de la Santísima Concepción, Chile

Redalyc: http://www.redalyc.org/articulo.oa?id=44057415010

anocetti@ucsc.cl

http://orcid.org/0000-0003-2509-8051

JoséLuis Medina-Moya

Universidad de Barcelona, España

jlmedina@ub.edu

(DD http://orcid.org/0000-0002-0487-9065

Recepción: 24 Febrero 2017

Aprobación: 04 Diciembre 2018

\section{Resumen:}

La política educativa chilena promueve la reflexión para mejorar la calidad de la formación docente. Resulta problemático que el cuerpo docente tenga dificultades para definir la reflexión y la utilicen como sinónimo de otros términos. El objetivo fue explorar la comprensión del significado que el profesorado en formación y sus formadores le dan a la reflexión pedagógica, profundizando en los vínculos que se establecen con el momento en el cual ocurre la reflexión y la racionalidad que la fundamenta. El enfoque metodológico fue interpretativo y se empleó un estudio de Casos Instrumental. Participaron 28 estudiantes de pedagogía en Biología quienes desarrollaban su práctica en la provincia de Concepción, seis formadores universitarios y 14 profesores guías de los centros de prácticas de los estudiantes. Se desarrollaron entrevistas en profundidad y focalizadas. Mediante análisis de contenido se observó el predominio de la reflexión entendida como proceso evaluativo, evidenciando una racionalidad técnica la cual separa la teoría y la práctica. Una minoría propone un significado deliberativo y no se encontró una noción crítica de reflexión. Se concluye que existe una vinculación entre la racionalidad del plan formativo, el significado de reflexión y el momento en que este proceso ocurre. Esto exige formadores universitarios y profesores guías que compartan una noción de reflexión y una actitud favorable hacia la reflexión práctica y crítica. Asimismo, se reconoce la importancia de revisar la racionalidad del plan de estudio, el cual podría constituir un obstáculo para que los profesores de biología en formación logren un mayor nivel de reflexión docente.

Palabras clave: Formador de docente, Práctica pedagógica, Docente en servicio, Reflexión.

\section{ABstract:}

Chilean educational policy promotes reflection to improve the quality of teacher training. Problems for teachers on defining reflection make them use it as synonym of other terms. The objective was to understand the significance that teachers in formation and their trainers give to pedagogical reflection, focusing on the links that are established with the moment when the reflection and the rationality that bases it, take place. The methodological approach was interpretive and a study of Instrumental Cases was used. 28 biology pedagogy students who developed their practice at the province of Concepción, 6 university trainers and 14 teacher guides from the students' practice centers, participated in the study. In-depth and focused interviews were developed. Through content analysis, the predominance of reflection as an evaluative process was observed, evidencing a technical rationality that separates theory and practice. A minority proposes deliberative significance, but any critical notion of reflection was found. It concludes that there's a link between the rationality of the training plan, the significance of reflection and the moment in which this process occurs. This requires university trainers and teacher guides who share a notion of reflection and a favorable attitude towards practical and critical reflection. Likewise, the importance of reviewing the rationality of the study plan is recognized, which could constitute an obstacle for biology teachers in training to achieve a higher level of teacher reflection.

KEYWORDS: Teacher educators, Teaching practice, School teachers secondary, Teacher in service, Reflection. 


\section{INTRODUCCIÓN}

La formación práctica se reconoce como un espacio relevante para la adquisición de conocimientos y habilidades la cual requiere el profesorado para cumplir con las exigencias actuales del ejercicio profesional. Esto, evidentemente, se favorece con una adecuada articulación entre el proceso formativo universitario y la experiencia que los estudiantes tienen en los centros de práctica. Entonces, el plan de estudio debe contemplar una reflexión sobre la acción, en que el aula escolar se constituye en un espacio para el aprendizaje docente.

Lo anterior cobra importancia cuando se quiere contar con profesionales reflexivos capaces de examinar su quehacer, problematizar y transformar su acción docente según las necesidades del alumnado y su contexto. Este desafío es difícil de concretarlo porque en las instituciones universitarias todavía se mantiene un enfoque formativo con un marcado acento técnico que constituiría una condición poco favorecedora del desarrollo de una postura reflexiva en el futuro profesorado.

Además, la noción de reflexión resulta ser problemática porque admite distintos significados, la reflexión según la perspectiva podría adoptar una perspectiva: filosófica, lingüística o pedagógica. Entonces, cuando se asume el reto de formar profesionales reflexivos debería considerarse la complejidad del concepto y sus implicancias metodológicas, tanto para estimular su desarrollo como para programas investigar los resultados de dichas propuestas formativas.

Cabe señalar que, a nivel institucional, se propuso la implementación de un modelo de práctica reflexiva que contempla: prácticas iniciales de inmersión, prácticas intermedias de intervención acompañada y luego autónoma. Se espera que, en los talleres universitarios, el alumnado examine su experiencia en los centros educacionales y a partir de esta, desarrolle sensibilidad pedagógica, una disposición indagadora para favorecer procesos de cuestionamiento y reconstrucción de los significados con que los estudiantes ingresan al programa de titulación. Lo anterior, evidentemente tiene como finalidad cultivar un saber que subraya la importancia de la relación pedagógica y exige un proceso de formación basado en un examen reflexivo habitual de lo que experimentan durante su estadía en la escuela.

$\mathrm{Al}$ inicio de la implementación del modelo de práctica reflexiva en la Facultad, interesó desarrollar una investigación cuyo objetivo fue comprender la experiencia de reflexión del estudiantado en uno de los programas de titulación, particularmente en la asignatura de práctica pedagógica profesional. Su importancia radicó en conocer desde los propios actores qué entendían por reflexión, explorar qué facilitaba o impedía el desarrollo de una práctica reflexiva y finalmente, entender como la experiencia reflexiva afectaba la identidad, la noción de lo pedagógico y lo didáctico en el profesorado en formación.

Este artículo tiene como objetivo explorar la comprensión del significado que el estudiantado y sus formadores le dan a la reflexión, profundizando en los vínculos que se establecen con el momento en el cual ocurre la reflexión y la racionalidad que la fundamenta. La importancia de los resultados reside en la identificación de la diversidad de significados de reflexión que circula entre el estudiantado y sus formadores. A partir, de ello se puede trabajar de modo consciente en un cambio de significado, según la racionalidad de la acción profesional que se quiera promover en el programa de titulación.

\section{ANTECEDENTES CONTEXTUALES Y ESTADO DE LA CUESTIÓN}

Uno de los desafíos actuales, en materia de política educativa nacional, es lograr formar profesionales reflexivos, lo cual resulta coherente con lo propuesto en la literatura internacional (Bárcena, 2005; Perrenoud, 2004). Se observa en el perfil de egreso de la mayoría de los programas de formación pedagógica la competencia reflexiva como un sello distintivo del profesional de la educación. No obstante a nivel nacional, de acuerdo a Tagle (2011) hay escasa evidencia que se haya logrado esta meta formativa.

La deseabilidad de una experiencia reflexiva se fundamenta en resultados de investigación reciente que evidencian una vinculación entre la reflexión y la disposición positiva hacia el mejoramiento de la práctica 
pedagógica (Correa, 2011; Tarrafello, 2006), su vínculo con el aprendizaje profesional, el reforzamiento de la identidad docente (Galaz, 2011) y la vocación (Nava y Reynoso, 2015; Nocetti, 2015). Asimismo, otros estudios establecen que favorece el examen de las propias creencias pedagógicas (Chacón-Corso, 2015) y la resignificación de la dimensión didáctica durante el desarrollo de la práctica pedagógica (Nocetti y Medina, 2018).

Otros estudios muestran que en la instituciones universitarias se desarrollan iniciativas para validar recursos y estrategias que estimulen procesos de reflexión durante la formación inicial de profesores (ChacónCorzo, 2008; Nocetti, 2016; Sanjurjo, 2012; Zabalza, 2005). Sin embargo, se percibe una falta de consciencia respecto de la complejidad del concepto, empleándolo sin suficiente precisión y de manera confusa en el campo de la educación (Beauchamp, 2006; Fendler, 2003).

Entonces, aunque se reconoce la importancia de desarrollar una práctica reflexiva durante la formación del profesorado, se evidencia insuficiente comprensión del concepto por parte del cuerpo formador (Lara, 2018). Esto resulta problemático porque dificulta el diseño y validación de estrategias de enseñanza y también, dificultad la descripción, comparación e interpretación de la experiencia reflexiva de los estudiantes durante su proceso formativo (Correa, Chaubet, Collin y Gervais, 2014).

En varias investigaciones se critica la diversidad de interpretaciones de la reflexión pedagógica y ponen de manifiesto la confusión que existe respecto de su significado (Beauchamp, 2015; Fendler, 2003). Se observa la utilización del concepto de modo especulativo (Beauchamp, 2006), es decir, no se explicita su significado, pudiendo conducir a errores de interpretación de resultados o llevar a conclusiones equivocadas.

Cabe precisar que Beauchamp (2006) determinó que el concepto de reflexión puede referirse a aspectos cognitivos, metacognitivos o lingüísticos. Asimismo, se asocia con el proceso de resolución de problema o con la crítica social (Correa et al., 2014). Otros autores suman la dimensión afectiva- emociones, sentimientoscomo un elemento central en la configuración de significado dicho proceso (Dewey, 1989; Lara, 2018; Nocetti, 2016; Schön, 1992).

Particularmente, en el campo de la educación el término de reflexión se utiliza como sinónimo de otros conceptos, tales como autoevaluación o metacognición. Tampoco se distingue la reflexión del pensamiento crítico, pero hay autores que precisan que el segundo concepto se refiere a la habilidad para evaluar afirmaciones y hacer juicios sobre la base de razones, en lugar de hacerlo basándose en hechos anecdóticos.

Finalmente, otro antecedente importante de considerar es que el discurso ministerial presente en documentos como el Marco para la Buena Enseñanza (Ministerio de Educación, 2003) o los Estándares Pedagógicos (Ministerio de Educación, 2012) evidencian una racionalidad técnica cuando se refieren a reflexión. Esto resulta ser una contradicción cuando a su vez esta entidad establece como meta formar profesores críticos reflexivos. Entonces, cabe preguntarse cómo afecta tal racionalidad el significado de reflexión que van configurando los docentes en servicio y el personal docente en formación.

\section{REFERENTES CONCEPTUALES}

En relación con la noción de reflexión, cabe señalar que el origen etimológico de este término deriva del latín reflectere que significa reflejar. También, puede ser definida como un acto de conciencia que posibilita el conocimiento sobre los propios actos y pensamientos (Ferrater, 1979). La reflexión puede concebirse como un tipo de pensamiento humano referido al "examen activo, persistente y cuidadoso de toda creencia o supuesta forma de conocimiento a la luz de los fundamentos que la sostienen y las conclusiones a las que se tienden" (Dewey, 1989, p.25).

Por su parte, Schön (1992) desarrolla la noción de práctica reflexiva, referida a un conocimiento que se construye a partir de la reflexión en y sobre la acción. Al extrapolar esta noción a la experiencia de los profesores, se podría afirmar que este conocimiento práctico reconfigura el marco de actuación de los profesores y además, mejora su práctica docente. Más recientemente, Perrenoud (2004) toma los conceptos 
de Schön (1992) y propone: la reflexión retrospectiva y prospectiva. La primera, hace alusión a la reflexión sobre la acción, siendo su función aprender sobre lo ocurrido y prepararse para una futura acción docente. Por su parte, la segunda se configura cuando se piensa en una acción docente futura, previendo como se actuará y relacionándose con una disposición favorable hacia la experimentación o indagación pedagógica.

Por otra parte, se suele utilizar el término de reflexión docente y de práctica reflexiva, como equivalentes. Pero, el segundo concepto se refiere a la reflexión que se experimenta de modo sistemático y habitual, formando parte del habitus del profesorado según los planteamientos de Schön (1998). Otro vocablo que tiende a usarse como sinónimo de la reflexión "es la reflexividad", el cual desde la perspectiva filosófica da cuenta de la capacidad de distanciarse del mundo y de sí mismo. Por lo anterior, Fendler (2003) plantea que el concepto de reflexividad no sería un objeto de estudio propio de la investigación educativa y debería reservarse exclusivamente para los estudios culturales. Los ejemplos anteriores, sin ser exhaustivos, evidencian la complejidad del significado de la reflexión como objeto de estudio, muestran la diversidad de enfoques para abordar su análisis, y además, ponen de manifiesto la dificultad que, en general, experimentan las personas para explicar qué entienden por este concepto.

El significado que se le atribuye a la reflexión puede estar influido por la racionalidad del modelo curricular que subyace a los planes de formación del profesorado. En esta perspectiva, es relevante exponer el planteamiento de Grundy (1987), quien basándose en la teoría de los intereses cognitivos de Habermas (1986) sugiere la existencia de tres tipos de racionalidad: una de carácter técnico encaminada al control, otra de tipo práctica orientada a la comprensión y finalmente, una racionalidad guiada por un interés crítico en el plan de formación del futuro personal docente. A continuación, se describe cada una de estas racionalidades:

Desde una racionalidad técnica y según Grundy (1987) los procesos formativos se focalizan en la imposición y reproducción de conocimientos en los estudiantes, proceso que no considera el contexto cultural y social. En esa perspectiva, se observa que se valora más lo teórico que la realidad de los sujetos (Pascual, 2011) y por consiguiente, el profesorado se transforma en un ejecutor de los programas que otros diseñan.

Por su parte, la racionalidad práctica acentúa una formación que privilegia la interacción con el espacio escolar para desarrollar habilidades de comprensión e interpretación del proceso educativo con criterios morales (Grundy, 1987). En este contexto, se valora el diálogo, el desarrollo del juicio y la reflexión se orienta a develar los significados que subyacen a la práctica educativa con el fin de deliberar lo que en ese contexto sería una buena acción (Pascual, 2011).En otras palabras, se desarrolla una acción ética que exige un juicio práctico por parte de los profesionales de la educación (Erazo, 2009).

La racionalidad crítica se distingue por el interés de promover en los procesos de formación una experiencia de emancipación de los sujetos, mediante el esclarecimiento de ideas o perspectivas deformadas de la realidad que significan desigualdad o exclusión social, entre otros (Grundy, 1987). En este contexto, cobra importancia las nociones de autonomía, autorreflexión y la praxis pedagógica.

El marco de interpretación anterior ha sido ampliamente utilizado para levantar tipologías de la reflexión (Hatton y Smith, 1995; Jay y Johnson, 2002; Larrivee, 2008) y en el campo de la noción de profesional reflexivo, destaca la propuesta de Van Manen (1977), quien establece niveles reflexivos que van desde el ejercicio de la reflexión basado en una racionalidad técnica hasta proponer una reflexión con una racionalidad crítica.

Varios autores (Domingo y Gómez, 2014; Erazo, 2009) han descrito la jerarquía propuesta por Van Manen (1977) en el ámbito del ejercicio de la docencia, planteando que la reflexión técnica devela el interés de utilizar el conocimiento educativo de modo efectivo y eficiente para lograr unos fines aceptados como dados. Por su parte, la reflexión práctica explicita los supuestos y predisposiciones presentes en la acción docente para valorar las consecuencias educativas hacia las que lleva esta. En este nivel el profesorado toma conocimiento de los fundamentos de su acción profesional y se incrementa el sentido valórico de su actuación. Finalmente, los 
profesores alcanzan una reflexión de carácter crítica cuando examinan su acción docente llegando a cuestionar las implicancias de su acción docente según criterios sociales, éticos y políticos.

\section{Procedimientos metodológicos}

De acuerdo al objetivo del estudio se desarrolló un estudio de carácter interpretativo (Álvarez, 2013; Crotty, 1998; Sandin, 2003) orientado a comprender desde la perspectiva de los sujetos el significado atribuido a la reflexión docente. De acuerdo a varios autores (Bisquerra, 2014; Flick, 2015; Rodríguez, Gil y García, 1999; Sandin, 2003) este tipo de investigación se vincula con el interaccionismo simbólico-focalizado y con a hermenéutica, interesada en la comprensión humana.

Se desarrolló un estudio de casos instrumental (Stake, 1998, 2013), en que el caso en sí mismo constituyó la base para profundizar en la comprensión del significado que los docentes en formación y sus formadores le atribuyen a la reflexión. Así, según Simons (2011) el estudio tuvo un carácter descriptivo situado en el contexto de la formación práctica, en un programa de formación de profesores de una universidad situada en la ciudad de Concepción al sur de Chile.

La relevancia de este caso obedeció a su potencial de transferencia, dado que este representa muchas de las características de otros programas de formación de profesores pertenecientes a la región del Biobío. En particular, se seleccionó la carrera de Biología porque desde el año 2014 estaba implementando un modelo de práctica reflexiva en la línea de formación práctica. De este modo, los resultados podrían servir a la propia institución al disponer de información respecto del tipo de significado de reflexión que está promoviendo mediante el plan de formación. Además, se estimó que esta experiencia podría constituir la base para examinar la experiencia de otros programas de formación con características similares a nivel local. En síntesis, los criterios de selección permitieron la comparación de la noción de reflexión según el tipo de práctica- pedagógica o profesional-, el tipo de centro de práctica- establecimiento público o privado, la formación del profesor universitario- pedagógica y/o disciplinar-, su experiencia docente para el caso de los profesores universitarios y de las escuelas o liceos.

Los informantes se conciben como aquellos sujetos que poseen una perspectiva que aporta a la comprensión del fenómeno que se está estudiando (Rodríguez et al., 1999; Valles, 2009), en este estudio los informantes fueron 28 estudiantes de pedagogía, seis profesores universitarios y 14 profesores en servicio.

Se utilizaron los siguientes criterios para su selección: En el caso de los estudiantes, debían pertenecer al programa de formación de profesores de Biología, estar cursando la asignatura de práctica pedagógica o profesional y desarrollando la práctica en una comuna con alto nivel de vulnerabilidad, tanto en un establecimiento público o particular subvencionado con bajos resultados académicos y que fueran parte de la provincia de Concepción. Los formadores universitarios debían contar con al menos cinco años de experiencia en docencia universitaria, particularmente, en taller de práctica pedagógica, con formación en el área de la educación y con especialización, en el área disciplinar de la Biología. En el caso de los profesores en servicio, estos debían cumplir el rol de profesores guías de los estudiantes, contar con mínimo cinco años de experiencia docente y trabajar en un establecimiento público o particular subvencionado perteneciente a la provincia de Concepción.

En importante hacer notar que se desarrolló muestreo teórico (Glaser y Strauss, 1968) para desarrollar las categorías, levantándose las dimensiones y atributos del significado de reflexión. De este modo, las decisiones respecto de las participantes se fueron tomando durante el trabajo de campo que se prolongó por dos años para ir profundizando en el significado que los distintos participantes le otorgaban a la noción de reflexión.

Se desarrollaron entrevistas en profundidad y entrevistas focalizadas (Coller, 2005; Gaínza, 2006; Simons, 2011; Valles, 2009) para lograr esclarecer el tipo de significado que los participantes del estudio le atribuían a la reflexión. Además, se exploraron relaciones entre significado y momentos en que se desarrolla habitualmente la experiencia reflexiva. Es importante considerar que todos los estudiantes han participado de 
una metodología implementada en el taller de práctica orientada a promover la reflexión docente mediante la observación de videos de clase a nivel individual y entre pares. Los datos cualitativos que emergieron de la conversación fueron analizados mediante un enfoque cualitativo, asumiendo la propuesta de Gibbs (2012) que incluyen las etapas de identificación de unidades de significado y la agrupación de categorías. Además, se contempló el cálculo de co-ocurrencia entre las categorías y la representación de estas relaciones apoyado por el programa de análisis cualitativo Atlas - ti 7.5.

\section{HALLAZGOS}

Se delimitaron un total de 489 códigos parte del núcleo temático denominado: Noción de la reflexión docente y elementos afines. En la Tabla 1 se puede observar la frecuencia con que se presentan estas unidades de significado.

\section{TABLA 1}

Tabla 1: Noción de la reflexión docente y elementos asociados

\begin{tabular}{|c|c|c|}
\hline Código & Categorias & Frecuencia \\
\hline SR_AU & $\begin{array}{l}\text { Significado de la reflexión_Descripción del significado de la reflexión con } \\
\text { énfasis en procesos de autoevaluación. }\end{array}$ & 58 \\
\hline SR_CS & $\begin{array}{l}\text { Significado de la reflexión_Descripción del significado de la reflexión con } \\
\text { énfasis en el cuestionamiento de la acción y creencias del docente. }\end{array}$ & 32 \\
\hline SR_PR & $\begin{array}{l}\text { Significado de la reflexión_Descripción del significado de la reflexión con } \\
\text { énfasis en procesos de problematización de la acción docente. }\end{array}$ & 20 \\
\hline PRPB & $\begin{array}{l}\text { Pregunta- causa problema. Descripción de preguntas centradas en las } \\
\text { causas del problema. }\end{array}$ & 18 \\
\hline PRCA & $\begin{array}{l}\text { Pregunta- consecuencia de mi acción. Descripción de preguntas centradas } \\
\text { en las consecuencias de la acción. }\end{array}$ & $\sqrt{3}$ \\
\hline PRIME & $\begin{array}{l}\text { Pregunta-mejoramiento de la acción docente. Descripción de preguntas } \\
\text { centradas en el mejoramiento de la acción docente. }\end{array}$ & 18 \\
\hline PRINE & $\begin{array}{l}\text { Pregunta-necesidades estudiantes. Descripcion de preguntas centradas en } \\
\text { las necesidades de los estudiantes. }\end{array}$ & 31 \\
\hline PRFU & $\begin{array}{l}\text { Pregunta -fundamentación. Descripción de preguntas centradas en la } \\
\text { fundamentación de la toma de decisiones y también de creencias. }\end{array}$ & 11 \\
\hline PRAU & $\begin{array}{l}\text { Pregunta Autoevaluación. Descripcion de preguntas centradas en la } \\
\text { evaluación del propio desempeño. }\end{array}$ & 49 \\
\hline PRDE & $\begin{array}{l}\text { Pregunta toma de decisiones. Descripcion de preguntas centradas en la } \\
\text { toma de decisiones. }\end{array}$ & 11 \\
\hline RE_AC & $\begin{array}{l}\text { Reflexión antes de la clase. Descripcion de procesos de reflexión que se } \\
\text { da durante la preparación de la enseñanza. }\end{array}$ & 43 \\
\hline RE_DC & $\begin{array}{l}\text { Reflexión durante la clase. Descripción de procesos de reflexion que se da } \\
\text { durante el desarrollo de las clases. }\end{array}$ & 75 \\
\hline$R E E_{-} F C$ & $\begin{array}{l}\text { Reflexion después de la clase. Descripción de procesos de reflexion que } \\
\text { se da después de la clase. }\end{array}$ & 82 \\
\hline CRD & $\begin{array}{l}\text { Causa de la reflexión docente. Descripción de las causas que } \\
\text { desencadena la reflexión en el contexto de la planificación de la } \\
\text { enseñanza, el desarrollo de la clase y al término de esta. }\end{array}$ & 35 \\
\hline RE_EIM & $\begin{array}{l}\text { Reflexion y emocion. Descripción de la emoción que está asociado al } \\
\text { proceso de reflexión docente. }\end{array}$ & 15 \\
\hline Total & & 489 \\
\hline
\end{tabular}

Fuente: Elaboración propia Unidad Hermenéutica Investigación Práctica, 2015. 
Tal como se describió anteriormente, a continuación se analizará el significado que le atribuyen a la reflexión el estudiantado de la asignatura de práctica profesional, docentes universitarios y el profesorado que cumple el rol de profesor guía en los centros educacionales.

\section{Subcategoría: Significado de reflexión como proceso de autoevaluación}

En los docentes en formación predominó la definición de reflexión como un proceso de autoevaluación. Para ellos y ellas reflexionar significó: detenerse y examinar sus acciones docentes con la finalidad de valorarlas según criterios establecidos en la institución formadora o escolar dónde desarrollan su práctica profesional.

Cuando digo reflexionar me refiero a mirar lo que uno hace y valorar lo que nosotros estamos haciendo correctamente o incorrectamente en nuestra práctica. Plantearnos críticamente, si lo estamos haciendo bien o lo estamos haciendo mal, de acuerdo a los estándares institucionales y también, desde lo que se dice en la teoría. Yo creo que es eso, ver los aspectos positivos y también, lo negativo que también tenemos, y así tomar decisiones para mejorar. (E2-2015.rtf- 5:20).

Este tipo de noción de reflexión se presentó habitualmente, al finalizar la sesión de clase de Biología y estuvo acompañada del deseo de mejorar la propia acción profesional futura, pero no necesariamente implicó un cambio de práctica.

O sea, yo solo reflexionaba después de la clase sobre lo que hice mal y pocas veces iba más allá. Evaluaba lo que hemos trabajado en la universidad para el inicio, el desarrollo y el cierre de la clase. En función de eso, uno piensa cómo mejorar la clase siguiente o al menos se lo propone porque a veces tampoco resulta. (Focus Group1-2015.rtf- 8:106).

Esta definición se relaciona con la propuesta de Dewey (1989), quien define el pensamiento reflexivo como un acto de distanciamiento con el propio pensamiento, en este caso, se trató de la acción docente. Por otra parte, este significado manifiesta un nivel bajo de reflexión en la jerarquía propuesta por Van Manen (1977), es decir, el estudiantado de pedagogía estaría refiriendo a una reflexión de carácter técnica. Estos resultados coinciden con otro estudio (Tagle, 2011), el cual sugiere a nivel nacional una baja reflexividad en el estudiantado en formación. Por su parte, Lara (2018), concluye que sentirse afectado emocionalmente y desear solucionar problemas pedagógicos, detonan reflexión de bajo nivel en el alumnado de pedagogía.

La reflexión como autoevaluación se presentó más habitualmente en estudiantes que desarrollaban su práctica profesional en establecimientos educacionales públicos de la comunidad local. Allí experimentaron el fracaso de sus propuestas didácticas, especialmente, al inicio de la práctica y cuando estaban por primera vez en este tipo de establecimiento.

Inicialmente, como que estaba más pendiente de mi desempeño porque era la práctica profesional y además, pocas veces había estado en un establecimiento municipal. Entonces, puedo decir que la reflexión era observar la efectividad de mi desempeño, especialmente, porque en ese centro de práctica nada me funcionaba como antes. Entonces, la reflexión yo la definiría como un ejercicio de autoevaluación. (E20- 2015.rtf-20-2).

Este resultado se conecta con dos ideas, por un parte, reafirma que la experiencia de reflexión tiene un carácter técnico en la mayoría del profesorado, representando un nivel bajo de desarrollo docente (Van Manen, 1997). Por otra parte, establece que la diversificación de espacios en la práctica estimula la reflexión por el potencial fracaso que experimentara el personal docente en formación (Nocetti, 2016).

Se encontró una co-ocurrencia de 0,33 entre este tipo de significado y la reflexión llevada a cabo al finalizar la clase. Se observó que esta relación estuvo más presente en el estudiantado que desarrollaba su práctica en establecimientos públicos caracterizados por su alta vulnerabilidad social. Cabe destacar, que la reflexión después de la clase se asoció con preguntas sobre el propio desempeño, observándose una co-ocurrencia igual a 0,30 entre ambas categorías en el discurso del futuro cuerpo docente de Biología que participó del estudio.

Porque después de finalizar una clase uno piensa ¿Qué hice bien? ¿Qué hice mal? Entonces es más profundo, no es como tan a la ligera $; Y$ a hago mi clase y listo! sino que uno igual se cuestiona hechos puntuales que haya pasado en la clase. (E4-2015.rtf $-18: 8)$. 
Esta reflexión al finalizar la clase se vincula con la noción de reflexión sobre la acción (Schön, 1992) y evidencia una racionalidad de carácter técnica, coincidiendo con otros estudios nacionales sobre procesos formativos que establecen lo mismo (Galaz, 2011; Labra, Montenegro, Iturra y Fuentealba, 2005; Latorre, 2003). Bajo esta racionalidad se valoran los resultados por sobre lo que ocurre en la sala de clases, reafirmando el carácter instrumental de las trayectorias formativas (Davini, 1995; Ferry, 1990). También, muestra una sobrevaloración de la aplicación de la teoría, sin suficiente examen de los significados que subyacen a la acción pedagógica (Davini, 1995; Grundy, 1987; Latorre, 2003; Russell, 2012; Sanjurjo, 2002).

También se exploró el significado en docentes universitarios del programa de formación pedagógica en Biología. La mayoría de ellos definieron la reflexión docente como un análisis de la propia acción, es decir, al igual que el profesorado en formación predominó un enfoque evaluativo y una racionalidad técnica.

La reflexión pedagógica para mí es como una especie de evaluación de mi actuación de acuerdo a ciertos criterios académicamente. Es poner en juicio el uso de estrategias y el nivel de efectividad logrado, por ejemplo, respecto de lo que se espera de una buena clase o de una actividad didáctica, en una clase de ciencias. (PU1-2015. .rtf - 63:6).

Este marcado acento instrumental permite comprender que la reflexión se focalice en la acción y no examine las intenciones, significados y consecuencias de la acción docente, determinando una reflexión posterior a la clase, a modo de control del producto de la acción profesional (Day, 2005; Perrenoud, 2004; Schön, 1992).

Los profesores de los centros educacionales reconocen que tienen dificultades para definir el concepto de reflexión y aunque manifiestan que es importante reflexionar, no saben cómo explicar su significado. En otras palabras, es un proceso que experimentan durante su trabajo, pero no saben cómo definirlo.

Reflexionar nos pide el ministerio. En todas partes está propuesto, en los documentos que recibimos, pero me es difícil definirlo. Además, tenemos tan poco tiempo para reflexionar nos reunimos en el departamento de ciencias, pero terminamos hablando de reglamentos, informando sobre nuestros resultados y la verdad, no hay un espacio para la reflexión. Tampoco, creo que sabemos muy bien cómo hacerlo. La verdad es un poco complicado el tema que me planteas, no sé bien cómo definirlo. (PG12- 2015. rtf-73:6).

Este hallazgo pone de manifiesto la falta de precisión con que se usa la noción de reflexión docente (Beauchamp, 2015) y como esto resulta problemático cuando se quiere avanzar hacia una práctica reflexiva, tanto en el estudiantado como en personal docente.

Finalmente, el profesorado definió la reflexión como un proceso de análisis de fortalezas, debilidades que te lleva a pensar en acciones docente alternativas. Así, se va constatando una alta coherencia entre el significado de formadores y profesorado en formación, en torno a una definición con un marcado enfoque evaluativo y un carácter instrumental.

La reflexión la veo como la identificación de mis errores, o pensar qué me falta para mejorar o qué podría haber respondido en un caso específico o qué podría haber hecho cuando reaccione mal. En el plano de la enseñanza, pensar ¿Cómo podría haber pasado un contenido otra forma? Es como una especie de autoevaluación de lo que uno hace. (PG11-2015. .rtf -72:4).

La experiencia formativa de la formación inicial docente no lleva al estudiantado a desarrollar altos niveles de reflexión según las propuestas de Van Manen (1977), Zeichner y Liston (1998) y Jay y Johnson (2002). Los resultados de este estudio se sumarían a la crítica de varios autores que ponen en cuestionamiento la posibilidad de formar profesionales críticos reflexivos, cuando los formadores evidencian confusión conceptual y en ellos predomina una reflexión con un carácter técnico (Beauchamp, 2015; Fendler, 2003; Korthagen, 2010; Russell, 2012)

Más recientemente se ha observado que este tipo de reflexión se vincula con la intención de cambio, pero no necesariamente con su concreción (Nocetti, 2016). Esto repercute sobre las oportunidades que tiene el futuro profesor de aprender a partir de los cambios que idea en y para la experiencia docente, tal como lo plantea Chacón-Corso (2015). 
Además, se observó reiteradamente que la noción de reflexión estaba centrada en la valoración del propio desempeño. Inicialmente, el profesor o profesora guía constituía el modelo a seguir, sin cuestionar necesariamente la pertinencia del modelo pedagógico que daba sustento a la propuesta didáctica de él o ella.

Yo al principio, me cuestionaba todo: ¿qué hice bien, ¿qué hice mal? y trataba de imitar la forma de enseñar de mi profesor guía. Yo creo que era para buscar su aprobación, pero con esto de la reflexión me empecé a cuestionar eso y empecé a hacer las cosas como yo sentía que salían mejor. Recuerdo que había harta presión en el establecimiento por cumplir con una serie de instrucciones que uno debía seguir, por ejemplo escribir el objetivo de la clase en la pizarra, o hacer una síntesis en el cierre. (Focus Group 4-2015.rtf-61:82).

\section{Subcategoría: Significado de reflexión como proceso de cuestionamiento}

En un grupo de estudiantes cambió el significado de reflexión durante el transcurso de la práctica. Plantearon que ya no se trataba de autoevaluación, sino del cuestionamiento que emerge del acto de observar la(s) consecuencia(s) de la propia actuación docente. En otras palabras, la reflexión deja de ser un proceso de autoevaluación y se transforma en un proceso de reflexión sobre la acción en la perspectiva de Schön (1998).

Cuando empezamos a trabajar con los videos, me empecé a preocupar más por el efecto de mi acción en los estudiantes. "Uno ve sus caritas y se da cuenta si funciona o no lo que uno está haciendo”. Antes como que pasaba de largo y ahora, uno se pone a pensar más en ellos durante la clase, yo lo conversaba con mis compañeras del taller, a todas nos ha pasado lo mismo. (Focus Group 1- 2015. rtf - 8:97).

En este caso, la reflexión entendida como cuestionamiento pudo estar influida por la metodología adoptada en el taller, que se centró en la observación de videos de clases entre compañeros. Varios autores reconocen cómo este recurso estimula la reflexión (Anijovich y Capelletti, 2014; Davini, 1995; Nocetti, 2016; Paquay y Wagne, 2010; Perrenoud, 2010).

Además, al centrarse en lo que sucede en la clase, a modo de una reflexión retrospectiva (Perrenoud, 2004), se acentúa el carácter deliberativo de la experiencia y por ello, la racionalidad que se manifiesta comienza a tener un carácter más práctico en la perspectiva de Van manen (1977). Entonces, no se produce solo un cambio de significado, sino que se acoge otra racionalidad (Grundy, 1987). Esto resulta relevante porque estaría evidenciando que el uso del video visibiliza al otro -el estudiantado- promoviendo una experiencia reflexiva con carácter práctico (Nocetti, 2016) y llevaría a ratificar que la reflexión docente puede ser estimulada mediante dispositivos específicos, tales como videos o escritura (Anijovich y Cappelletti, 2014).

Pareciera que este grupo de estudiantes se logró una mayor comprensión de las necesidades del estudiantado y su contexto. El objeto de la reflexión deja de ser el sí mismo, para centrarse en el otro: el estudiantado. También, cambió el momento en el cual se produce la reflexión, incrementándose la reflexión durante el desarrollo de las clases. Varios estudiantes de pedagogía en Biología reconocieron que la reflexión durante la clase comenzó hacerse más habitual. De este modo, se observa con claridad el vínculo entre el cambio de significado, de racionalidad y el momento en que se crea la reflexión docente.

A mí me pasó algo similar, como que las prácticas anteriores yo reflexionaba más al finalizar la clase. En cambio, ahora me cuestioné las cosas dentro de la misma clase, observé la reacción de los estudiantes y dudé sobre lo tenía preparado, sentí la necesidad de cambiar mucho la planificación. Al inicio de la práctica como que todo era más mecánico y la planificación mandaba. (Focus Group 1- 2015.rtf - 8:87).

Se observó que la reflexión que lleva a reconocer consecuencias de la propia acción docente incrementa la habilidad para cambiar mientras se desarrolla la clase de Biología. El análisis de videos entre pares aumentó la comprensión de lo que significa enseñar, identificándose aspectos de la enseñanza que debían cambiar para responder mejor a las necesidades del estudiantado. Lo anterior, denota el potencial de aprendizaje que tiene la reflexión en la acción (Schön, 1992) y en este caso se presenta unida a un cambio de racionalidad de carácter práctica (Grundy, 1987).

Yo creo que la reflexión es un cuestionamiento de lo que uno hace. A mí me ha pasado que llego a mí clase, veo la reacción del estudiantado y pienso en otra cosa y cambio en el instante. Ahora estoy más atenta a lo que sucede en la clase y tengo 
más confianza para hacer los cambios y esto lo he aprendiendo, especialmente, en este establecimiento de carácter público. (Focus Group 2-2015.rtf- 9:37).

Se pudo establecer mediante análisis de co-ocurrencia, que existe vinculación entre la reflexión que se produce durante la clase y el cambio en el modo de hacer la clase, alcanzando un valor de 0,30. Los resultados permiten suponer que la reflexión que se da durante la acción docente se asocia con cambios de la enseñanza que se implementan en el curso de la clase misma y por lo tanto, se enriquece el conocimiento en la acción de acuerdo a los planteamientos de Schön (1998).

Los profesores y las profesoras guías plantean que la experiencia docente aumenta la habilidad para realizar cambios durante la clase, aprendiendo de lo que acontece en el aula. Una profesora comentó su experiencia de reflexión durante el desarrollo de una clase:

Creo que a la mayoría de los profesores que tienen varios años de experiencia cuando un contenido no resulta del interés del estudiantado. Entonces, uno se detiene y hace un cambio. Uno se pregunta: ¿Cómo los intereso? O trata de buscar una experiencia que sea interesante para ellos y ellas. Algo que llame su atención y cambia de actividad. Con el tiempo se desarrolla cierta confianza para cambiar lo planificado mientras trascurre la clase. (PG5-2015. rtf- 70:10).

Una minoría de estudiantes presentó una noción de reflexión entendida como el examen de creencias o supuestos que fundamentan la acción docente. Este significado se acentuó en el personal docente en formación que mantenía estrechas relaciones con el estudiantado. Esta experiencia coincide con el planteamiento de (Korthagen, 2010), quien afirma que a mayor oportunidad para mejorar las relaciones interpersonales con el estudiantado en la escuela, más se incrementa la reflexión docente con vista a un mejoramiento de la práctica.

También, creo que reflexionar es cuestionar esas creencias sobre la enseñanza que uno tiene tan internalizado. Cuando uno toma conciencia del contexto social donde está, entonces empieza a revisar sus creencias y los fundamentos de su acción profesional. Yo creo que ese fue uno de mis mayores desafíos porque si uno cambia la forma de pensar la acción docente, también cambia el modo de estar en aula y puede responder mejor a los desafíos sociales que te plantea un establecimiento educacional como este. (E9-2015.rtf - 52:29).

Se compararon las experiencias de cada practicante en distintos tipos de escenarios escolares y se observó que la definición de reflexión centrada en el cuestionamiento de las creencias está más presente en estudiantes que desarrollaron su práctica en establecimientos de carácter públicos. Pareciera que esto se explica porque en dichos escenarios se produce un mayor desequilibrio de carácter práctico en palabras de Nocetti y Medina (2018).

Para mí la reflexión es la mirada hacia el interior, reconocer qué sentido le doy a la enseñanza, pero honestamente. En el fondo examino lo que pienso y me doy cuenta que muchas ideas que aprendemos en la universidad pierden su validez en el aula escolar real. Surge la necesidad de darle otro sentido a la práctica docente y creo que esto se da cuando uno mira la profesión más allá del trabajo dentro del aula, reconociendo la dimensión social esta. (E13-2015.rtf - 20:11).

La experiencia en las escuelas aumentó el fracaso en el profesorado en formación, favoreciendo un mayor cuestionamiento de la propia práctica. Así, el conjunto de practicantes asumió que dicha experiencia acentuó la revisión de sus creencias sobre lo pedagógico, didáctico, según lo acontecido en la sala de clases real. Esto reitera la importancia de que desarrollen estadías en escenarios escolares diversos y desafiantes, en que enfrenten el fracaso de sus actividades didácticas (Nocetti y Medina, 2018). Además, pone en evidencia la importancia de estadías prolongadas, favoreciendo una estrecha relación con el alumnado. Esto promueve que se sientan afectados emocionalmente (Lara, 2018) para acentuar así la reflexión.

Experimente dudas sobres mis creencias a raíz de mi práctica en este liceo vulnerable. Antes no me preguntaba sobre el sentido de la educación, ahora, me lo cuestiono todo. El mundo del liceo es muy distinto a otros centros educacionales y cambiaron mis ideas sobre la educación y también, mis prioridades. (E21-2015.rtf-30: 15). 
En general el profesorado en formación, piensa que la teoría aprendida en la universidad, pierde validez en la escuela, incrementándose el cuestionamiento sobre el discurso trasmitido por la institución formadora. Esto está reclamando una mayor articulación entre la teoría y la práctica, en el contexto del proceso de formación inicial de profesor de Biología (Korthagen, 2010). Esto exige mayor trabajo colaborativo entre la universidad y los centros escolares (Hirmas y Cortés, 2015).

A mí me tocó enfrentar algunas barreras respeto del marco teórico con que uno va al liceo. Aquí las cosas funcionan de modo diferente y el fracaso te hace dudar sobre las creencias que tienes. Uno se lo cuestiona todo y además, va cambiando el modo de entender la enseñanza. (FOCUS GROUP 1- 2015-.rtf - 8:4).

Docentes en formación reconocieron que la reflexión entendida como cuestionamiento de las creencias fue más habitual durante la preparación de la enseñanza, es decir, el conocimiento paulatino de las características del estudiantado, llevó a deliberar durante la preparación de las clases que sería una clase más provechosa para tal alumnado.

La teoría se derrumba en los colegios y eso provoca frustración. Los profesores de la universidad se centran mucho en realidades de otro tipo, pero cuando uno lleva todo eso que te enseñaron al liceo, y te das cuenta de que esa realidad es totalmente distinta aumentan las dudas sobre el sentido de tu profesión. (P47: E2- -2015.docx - 47:15).

\section{Subcategoría: Significado de reflexión como proceso de problematización}

Al finalizar la asignatura de práctica, algunos estudiantes, definen la reflexión como un proceso de problematización de su quehacer docente.

En esta práctica con la metodología de los videos, uno problematiza más lo que hace. El video te hace reflexionar permite ver a los estudiantes y se identifican problemas de tu quehacer docente que hay que mejorar. La reflexión deja de ser como antes una autoevaluación, como que ahora se hizo más evidente la problematización y la búsqueda de una solución. (Focus Group 6-2015. rtf-61:15).

El cuerpo docente en formación señaló que cuando la reflexión es concebida como un proceso de problematización, se movilizan acciones de experimentación / indagación, encaminadas a superar la situación que se percibió como una problemática. Esto coincide con el planteamiento de varios autores, quienes plantean que la reflexión problematiza, mejorando la habilidad para delimitar problemas y encontrar soluciones alternativas a las tradicionales (Korthagen, 2010; Schön, 1998).

Este significado de reflexión se vincula con la imagen de un profesor que analiza su propia práctica, la problematiza y trabaja para mejorar en función de lo que necesitan sus estudiantes, denotando una racionalidad de carácter práctico (Grundy, 1987).

Nunca antes se dio lo de la reflexión así, en las prácticas anteriores, yo hacía siempre todo del mismo modo, la misma metodología, las mismas explicaciones. Pero, ahora el tema era identificar un problema en mi propia práctica y trabajar en ello, y eso me obligó a reflexionar cómo lograr un cambio. Esa experiencia cambió el significado de reflexión y ahora la concibo como un proceso de problematización que culmina en la concreción de un cambio en el aula. (E18-2015 .tf- 48:3).

Se encontró una vinculación entre la concepción de reflexión como problematización y los cambios implementados durante la clase. El índice de co-ocurrencia alcanzó un valor de 0,30 y de este modo, se devela que este tipo de significado está más presente en estudiantes que logran incorporar cambios en su práctica pedagógica durante el curso de la acción docente.

Además, aquí fue notorio como la reflexión problematiza al punto que uno siente descontento, busca apoyo en la teoría para encontrar una solución. Ideamos un plan, lo implementamos y observamos los resultados. Entonces se une la reflexión y cambio, se siente el aprendizaje basado en lo que se hace para cambiar. (E27-2015.rtf - 20:25).

Un aspecto importante a recalcar es que en la asignatura de Práctica Profesional el propio estudiante identificó el problema que decidió resolver, tal como se describe a continuación: 
Difícil fue decir esto constituye mi problema es que estamos acostumbrados a que nos digan: "este es tu problema”. Yo creo que fue clave que cada uno definiera su problema y no el profesor. El compromiso con el cambio fue mayor, es tu elección a raíz del descontento y uno siente que debe solucionarlo sí o sí. (E28-2015.rtf - 47:10).

Los estudiantes reconocieron que los compañeros fueron claves en el proceso de reflexión entendida como problematización. Estos permiten aclarar cuál es realmente el problema o esclarecer problemas no advertidos. Esto explicaría la importancia de promover experiencias de reflexión entre pares durante la formación inicial, reconociendo la dimensión social de la práctica reflexiva. Además, los resultados de varias investigaciones demuestran un aumento en la efectividad de la acción docente cuando los profesores problematizan su práctica en conjunto con otros colegas.

Cuando analicé mi clase con mis compañeros, me ayudaron a identificar mi problema. A veces uno de nosotros dice: "este es mi problema”, pero no, porque el problema tal vez es otro y quizás la solución no sea esa. Los compañeros te cuestionan a veces más profundo y te dejan ver de otra forma lo que aparece en el video. (Focus Group 3- 2015.rtf - 8:10).

La diversidad de significados de reflexión encontrados pone de manifiesto la complejidad de la noción de reflexión (Fendler, 2003; Beauchamp, 2015) y la importancia de explorar la experiencia de los actores en contexto formativo, coincidiendo con Ruffinelli (2017), quien propone que todavía hay dificultades para identificar modelos efectivos para desarrollar una reflexión pedagógica sistemática.

También, lleva a reconocer la importancia del papel de la práctica pedagógica como un espacio para ejercitar el desarrollo de esta habilidad de modo práctico y crítico, tal como lo plantea Tagle (2011). Cuidando antes consensuar entre el cuerpo formador el significado de reflexión y la racionalidad que se quiere estimular.

Finalmente, considerando los niveles de reflexión propuestos por Van Manen (1977), se propone una clasificación del significado de reflexión docente. En síntesis, la reflexión puede ser definida como un proceso de autoevaluación, cuestionamiento de creencias y una problematización de la acción profesional. En la Tabla 2 se presenta la clasificación del significado de la reflexión docente que emergió de los participantes de este estudio. 
TABLA 2

Tabla 2: Clasificación del significado de la reflexión según la racionalidad

\begin{tabular}{|c|c|c|c|}
\hline Racionalidad & $\begin{array}{l}\text { Significado de } \\
\text { Reflexión }\end{array}$ & Ejemplo de citas & Frec. \\
\hline 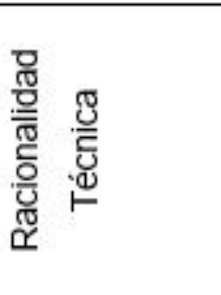 & $\begin{array}{l}\text { Reflexión como } \\
\text { Autoevaluación }\end{array}$ & $\begin{array}{l}\text { O sea, para mi reflexionar es observar lo } \\
\text { que se está haciendo bien y mal. Además, } \\
\text { pocas veces va más allá, es como un } \\
\text { plano de autoevaluación. Focus Group. 1- } \\
2015 . r t f-8.24\end{array}$ & 58 \\
\hline \multirow{2}{*}{ 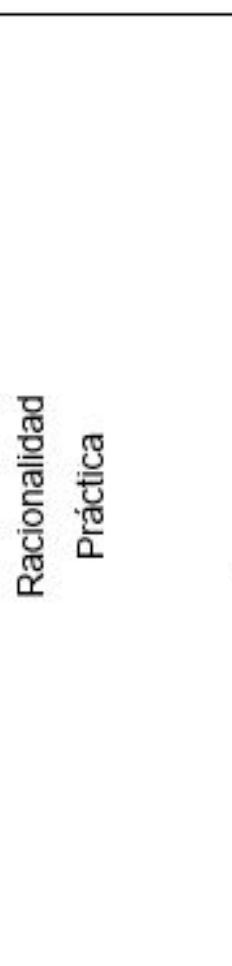 } & $\begin{array}{l}\text { Reflexión como } \\
\text { cuestionamiento de } \\
\text { la acción }\end{array}$ & $\begin{array}{l}\text { Para mi la reflexión docente, su } \\
\text { significado es como un cuestionamiento } \\
\text { que el docente se plantea sobre la labor } \\
\text { que cumple dentro de la sala de clase, } \\
\text { para lograr responder a las necesidades } \\
\text { de los estudiantes y considerar cambios } \\
\text { en el caso de que sea necesario. Es poner } \\
\text { en duda lo que se hace y se piensa. Focus } \\
\text { Group. 5- 2015.docx - } 61: 1\end{array}$ & 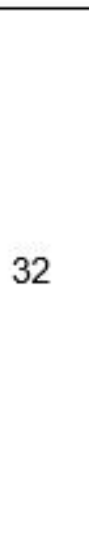 \\
\hline & $\begin{array}{l}\text { Reflexión como } \\
\text { problematización }\end{array}$ & $\begin{array}{l}\text { Yo creo que la reflexión la viví primero } \\
\text { como una evaluación, pero luego la } \\
\text { reflexión fue como una problematización. } \\
\text { Porque primero lo más básico es que uno } \\
\text { se autoevalúa lo que está haciendo bien o } \\
\text { lo que está haciendo mal, casi } \\
\text { inconscientemente. E10-2015.rtf-20:25 }\end{array}$ & 20 \\
\hline Total & & & 110 \\
\hline
\end{tabular}

Fuente: Elaboración propia, Unidad Hermenéutica Investigación Práctica, 2015.

\section{ConcLusión}

Conforme al objetivo del artículo y luego de presentar los resultados, se puede afirmar que no existe un único significado para la reflexión en docentes en formación del programa de pedagogía en Biología y sus formadores. Se observaron tres significados: la reflexión como proceso de autoevaluación, de cuestionamiento de la acción y de problematización.

El significado que predominó en el estudiantado y sus formadores fue una noción de reflexión entendida como un proceso de autoevaluación. Esto permite proponer la presencia de un esquema conceptual compartido que evidentemente se reproduce y obstaculizaría la configuración de otros significados para la reflexión que sobrepasen un enfoque evaluativo.

Se podría plantear que el grupo formador puede constituir una barrera para que la noción de reflexión se desarrolle en el estudiantado. Esto resulta problemático en varios sentidos, por una parte, significa que reflexionar se limitaría a emitir un juicio sobre la propia práctica, pero no necesariamente garantizaría un 
cambio de esta. Por otra parte, este marco conceptual compartido disminuye las opciones de mejoramiento de la propia acción profesional, cuestión que normalmente es asociada a la práctica reflexiva.

Solo una minoría de estudiantes y docentes formadores definió la reflexión como un proceso de problematización de la propia práctica. Esto permite afirmar que, aunque participen de experiencias reflexivas, la mayoría mantiene una comprensión técnica del significado de reflexión, la que pondría en riesgo la factibilidad de lograr una formación de profesionales reflexivos capaces de comprender, deliberar y desarrollar mejoras sistemáticas a su actuación profesional.

No obstante lo anterior, los hallazgos permiten concluir que la noción de reflexión puede transformarse. En este caso, la observación de videos de clases estimuló un significado de reflexión centrado en el cuestionamiento de la propia práctica a partir de lo que le sucede al otro: el alumnado.

El hallazgo anterior, permite recomendar la selección intencional de estrategias- observación, escritura o diálogo entre pares- que estimulen el examen de las consecuencias de la propia actuación docente, abriéndose a la deliberación para decidir qué significa una buena acción profesional en un contexto particular.

La falta de explicitación y consenso respecto del significado de reflexión entre el cuerpo de formadores, obstaculiza el diseño de experiencias formativas y su evaluación. Por consiguiente, el valor de estos resultados radica en que hay escasas investigaciones nacionales que ponen de manifiesto esta problemática y que evidencian que el significado que estos le dan a este concepto podría constituirse en un factor obstaculizador del desarrollo de una postura reflexiva en el futuro personal docente. Por consiguiente, no se puede afirmar que adoptar un enfoque reflexivo es garantía de profesionalización, todo depende de la coherencia entre el significado y la racionalidad que se logre en el plan de estudio.

En el contexto anterior, convendría reconocer que la reflexión es un medio para mejorar la acción profesional y no el fin en sí mismo, cuestión que no se ha discutido suficientemente en los programas de formación pedagógica a nivel nacional. Además, la similitud de significado entre el profesorado en formación y docentes formadores resulta preocupante, porque cuando quienes están a cargo del proceso evidencian un predominio de la reflexión con un carácter técnico es improbable que el estudiantado sobrepase esta noción de reflexión.

En relación con la racionalidad que subyace en el significado que los participantes le otorgan a la reflexión, se puede concluir que predomina una racionalidad técnica, coincidiendo con el significado de la reflexión como un proceso de autoevaluación. Esto significa que se instrumentaliza la acción docente y se valoran más los resultados, en desmedro del examen del proceso que ocurre en la sala de clases. Además, se evidencia una concepción formativa que sobrevalora la teoría y su aplicación, sin suficiente espacio para el aprendizaje desde una práctica reflexiva basada en el desarrollo del juicio deliberativo del profesorado.

Este marcado acento instrumental permite comprender que la reflexión se focalice en la efectividad de la acción y no preste suficiente atención a los valores y significados que subyacen a la acción docente. Pareciera que para sobrepasar la racionalidad técnica es necesaria una mayor colaboración entre el mundo universitario y el ámbito de los establecimientos educacionales. El profesor guía se constituiría en un co-formador y la sala de clase en un campo para la experimentación, indagación y la construcción de conocimiento pedagógico.

En esta investigación, se podría plantear que el examen de la propia acción-mediada por videos-, no solo cambió el significado otorgado por el estudiantado a la reflexión, sino también la racionalidad, la que se inclinó hacia una postura práctica como base de la acción profesional.

Al final, no se visibilizó una racionalidad crítica en el significado de reflexión, pudiendo ser la expresión del paulatino alejamiento del profesorado de temas sociales y políticos. Por consiguiente, este hecho evidencia el efecto de condiciones estructurales en la que están insertos los docentes. Entonces, se necesitan experiencias formativas que desarrollen una postura crítica frente a la propia actuación docente, develando suposiciones, ideas o significados que atentan contra la igualdad de oportunidades en el acceso a la educación o generan exclusión permanente de las minorías o del estudiantado que representa los márgenes de la escuela. 
El estudio tiene relevancia a nivel de la carrera porque invita a revisar la racionalidad del plan de estudio en materia de formación práctica y consensuar un significado para la reflexión pedagógica entre el grupo de formadores. Además, pone de manifiesto la necesidad de asumir la tarea de desarrollar procesos formativos tempranos que estimulen una reflexión con carácter crítico, devolviendo al ejercicio profesional su dimensión ética, política y social.

Finalmente, cabe señalar que el muestreo teórico desarrollado limitó la discusión en términos de categorías como género. Además, los resultados que aquí se presentan no pueden generalizarse a otros programas de formación. Sin embargo, se espera que los hallazgos que se exponen motiven la exploración del significado de reflexión y la racionalidad que caracteriza el enfoque curricular en el campo de la formación práctica, a fin de tomar los resguardos para garantizar el logro de la formación de profesionales reflexivos.

\section{REFERENCIAS}

Álvarez, J. (2013). Cómo hacer una investigación cualitativa. Fundamentos y metodología. México: Paidós.

Anijovich, A. y Cappelletti, G. (2014). Las prácticas como eje de la formación docente. Argentina: Universitaria de Buenos Aires.

Bárcena, F. (2005). La experiencia reflexiva en la educación. Barcelona: Paidós.

Beauchamp, C. (2006). Understanding Reflection in Teaching: A Framework for Analyzing the Literature (Thesis Doctor). McGiII University, Montreal, Canadá. Recuperado de https://bit.ly/2SXGCnp

Beauchamp, C. (2015). Reflection in teacher education: issues emerging from a review of current literature. Reflective Practice. International and Multidiciplinar y Perspectives, 16(1), 123-141.

Bisquerra, R. (2014). Metodología de la investigación educativa. Madrid: La Muralla.

Chacón-Corzo, M. (2008). Las estrategias de enseñanza reflexiva en la formación inicial docente. Educere, 12(41), 277-287.

Chacón-Corzo, M. (2015). La construcción del conocimiento sobre la enseñanza desde la perspectiva de los futuros docentes. Revista Educación, 39(1), 51-67. Recuperado de https://goo.gl/aCXQsw

Coller, X. (2005). Estudio de casos. Madrid: Centro de Investigaciones.

Correa, E. (2011). La práctica docente: Una instancia de desarrollo profesional. Perspectiva Educacional, 50(2), 77-95.

Correa, E., Chaubet, P., Collin, S. y Gervais, C. (2014). Desafíos Metodológicos para el estudio de la reflexión en contexto de formación de profesores. Estudios Pedagógicos, 40(1), 71-86.

Crotty, M. (1998). The foundations of social research: Meaning and perspective in the research process. London: Sage.

Davini, M. (1995). La formación docente en cuestión: política y pedagogía. Buenos Aires: Paidós.

Day, C. (2005). Formar docentes. Cómo, cuándo y en qué condiciones aprende el profesorado. Madrid: Narcea.

Dewey, J. (1989). Cómo pensamos. Nueva exposición de la relación entre pensamiento reflexivo y proceso educativo. Barcelona: Paidós.

Domingo, A. y Gómez, M. (2014). La práctica reflexiva. Bases, modelos e instrumentos. Madrid: Narcea.

Erazo, S. (2009). Práctica reflexiva como estrategia de desarrollo profesional: presencia y estructura en reuniones docentes. Educador y Educadores, 12(2).

Fendler, L. (2003). Teacher reflection in a hall of mirrors: historical influences and political reserberations. Educational Researcher. 32(3), 16-25.

Ferry, G. (1990). El trayecto de la formación. Los enseñantes entre la teoría y la práctica. México: Paidós.

Ferrater, J. (1979). Diccionario de filosofía, 1. Madrid: Alianza.

Flick, U. (2015). Diseño de la investigación cualitativa. Madrid: Ediciones Morata.

Gaínza, Á. (2006). La entrevista en profundidad individual. En: M. Canales Cerón (Ed.), Metodologías de investigación social: Introducción a los oficios (pp. 219-263). Santiago, Chile: LOM. 
Galaz, A. (2011). El profesor y su identidad profesional: ¿facilitadores u obstáculos del cambio educativo? Estudios Pedagógicos, 37(2), 89-107

Gibbs, G. (2012). El análisis de datos cualitativos en la investigación cualitativa. Madrid: Morata.

Glaser, B. y Strauss, A. (1968). The discovery of grounded theory: Strategies for qualitative research. London: Weidenfeld and Nicolson.

Grundy, S. (1987). Producto praxis del Currículum. Madrid: Morata.

Habermas, J. (1986). Conocimiento e interés. Taurus. Madrid.

Hatton, N. y Smith, D. (1995). Reflection in teacher education: Towards definitions and implementation. Teaching and Teacher Education, 11, 33-49.

Hirmas, C. y Cortés, I. (2015).Investigaciones sobre la formación práctica en Chile: Tensiones y desafíos. Santiago de Chile: Organización de Estados Iberoamericanos para la Educación, la Ciencia y la Cultura.

Jay, J. K. y Johnson, K. L. (2002). Capturing complexity: A typology of reflective practice for teacher education. Teaching and Teacher Education, 18(1), 73-85.

Korthagen, F. (2010). La práctica, la teoría y la persona en la formación del profesorado. Revista Interuniversitaria de Formación del Profesorado, 24(2), 83-101.

Labra, P., Montenegro, G., Iturra, C. y Fuentealba, R. (2005). La investigación acción como herramienta para lograr coherencia de acción en el proceso de práctica profesional durante la formación inicial docente. Estudios Pedagógicos, 31(2), 137-143.

Lara, B. (2018). Análisis de significados de la reflexión pedagógica de profesores en Formación inicial. Revista de estudios y Experiencias en educación, 17(33), 101-111.

Larrivee, B. (2008). Development of a tool to assess teachers' level of reflective practice. Reflective Practice, 9(3), 341360.

Latorre, M. (2003). Aportes para el análisis de las racionalidades presentes en las prácticas pedagógicas. Revista Iberoamericana de Educación, 36(2), 58-72.

Ministerio de Educación (2003). Marco para la Buena Enseñanza. Santiago, Chile: CPEIP.

Ministerio de Educación (2012). Estándares orientadores para carreras de pedagogía en educación media. Santiago, Chile: Lom Ediciones.

Nava, G. y Reynoso, J. (2015). Conceptualización y reflexión sobre la práctica educativa en un programa de formación continua para docentes de educación media superior en México. Revista Educación, 39(1), 137-157. Recuperado de https://goo.gl/MkYHv1

Nocetti, A. (2015, octubre). El ciclo de Aprendizaje Reflexivo como dispositivo para estimular la reflexión y el cambio docente en la práctica profesional. VIII Jornadas nacionales y I Internacional sobre la Formación del Profesorado. Narrativa, Investigación y reflexión sobre las prácticas. Universidad Nacional de Mar del Plata, Argentina. Recuperado de https://goo.gl/AKwusM

Nocetti, A. (2016) Facilitadores de reflexión docente durante la práctica profesional en escuelas vulnerables de Concepción, Chile. Educadi, 1(1), 83-100. Recuperado de https://goo.gl/neSdxZ

Nocetti, A. y Medina, J. (2018). Condiciones que desencadenan la reflexión docente en el futuro profesor durante sus prácticas de formación. Revista Espacios, 39(15).

Pascual, E. (2011). Racionalidades en la producción curricular y el proyecto curricular. Pensamiento Educativo. Revista de Investigación Educacional Latinoamericana, 23(2), 13-72.

Paquay, L. y Wagner, M. (2010). Formación continua y video formación: qué habilidades se deben priorizar. En: L. Paquay, M. Altet, E. Charlier y P. Perrenoud (Coords) La formación profesional del maestro. Estrategias y Competencias (pp. 222-263). México: Fondo de Cultura Económica.

Perrenoud, P. (2004). Desarrollar la práctica Reflexiva en el oficio de enseñar. Barcelona: Graó.

Perrenoud, P. (2010). El trabajo sobre el habitus en la formación de maestros. Análisis de las prácticas y toma de conciencia. En: L. Paquay, M. Altet, E. Charlier y P. Perrenoud (Coords). La formación profesional del maestro. Estrategias y Competencias (pp. 265-304). México: Fondo de Cultura Económica. 
Rodríguez, G., Gil, J. y García, E. (1999) Metodología de la Investigación Cualitativa. España: Aljibe.

Ruffinelli, A. (2017). Formación de docentes reflexivos: un enfoque en construcción y disputa. Educación y Pesquisa, 43(1), 97-111.

Russell, T. (2012). Cambios paradigmáticos en la formación de profesores: Peligros, trampas y la promesa no cumplida del profesional reflexivo. Rencontres on Education, 13, 71-91.

Sandin, M. (2003). Investigación cualitativa en educación. Fundamentos y tradiciones. Madrid: Mac-GrawHill.

Sanjurjo, L. (2002). La Formación práctica de los docentes. Reflexión y acción en el aula. Buenos Aires: Homo Sapiens.

Sanjurjo, L. (2012) Razones que fundamentan nuestra mirada acerca de la formación práctica. En L. Sanjurjo, A. Caporossi, A. España, I. Alfonso y M. Foresi (Coords). Los dispositivos para la formación en las prácticas profesionales (pp. 15-43). Argentina: Homo Sapiens.

Schön, D. (1992). La formación de profesionales reflexivos. Hacia un nuevo diseño de la enseñanza y el aprendizaje en las profesiones. Barcelona: Paidós.

Schön, D. (1998). El profesional reflexivo. Cómo piensan los profesionales cuando actúan. Barcelona: Paidós.

Simons, H. (2011). El estudio de caso: teoría y práctica. Madrid: Morata.

Stake, R. (1998): Investigación con estudio de casos. Madrid: Morata.

Stake, R. (2013). Estudios de casos cualitativos. En: N. Denzin y. Lincoln (Coords.). Las estrategias de investigación cualitativa (pp. 154-197). España: Gedisa.

Tagle, T. (2011). El enfoque reflexivo en la formación docente. Calidad en la Educación, 34, 203-215.

Tarrafello, D. (2006). La formación para la práctica reflexiva en las prácticas profesionales docentes. Educere, 10(33), 269-273.

Valles, M. (2009). Entrevistas cualitativas. Cuadernos Metodológicos. España: CIS.

Van Manen, M. (1977). Linking ways of knowing with ways of being practical. Curriculum Inquiry, 6(3), 205-208.

Zabalza, A. (2005). Diarios de Clase. Un instrumento de investigación y desarrollo profesional. Madrid: Narcea.

Zeichner, K. y Liston, D. (1998). Formando profesores reflexivos. En A. Alliaud y L. Duschatzky (Comp.). Maestros. Formación, práctica y transformación escolar (pp.263- 297). Buenos Aires: Miño y Dávila.

\section{BY-NC-ND}

